\title{
Endoscopic Ultrasound-Guided Portal Pressure Measurement and Interventions
}

\author{
Jason B. Samarasena and Kenneth J. Chang \\ Division of Gastroenterology and Hepatology, H. H. Chao Comprehensive Digestive Disease Center, University of California, Irvine Medical \\ Center, Orange, CA, USA
}

A growing number of studies have explored endoscopic ultrasound (EUS)-guided vascular catheterization. Potential clinical applications of EUS-guided portal venous access include angiography, measurement of the portosystemic pressure gradient, EUS-guided transhepatic intrahepatic portosystemic shunt creation and portal vein sampling for the evaluation in gastrointestinal cancer. The following article reviews the different devices and techniques employed in these applications. Clin Endosc 2018;51:222-228

Key Words: Endosonography; Portal pressure gradient; Hepatic venous portal gradient; Portal vein sampling; Transhepatic intrahepatic portosystemic shunt

\section{INTRODUCTION}

A growing number of studies have explored endoscopic ultrasound (EUS)-guided vascular catheterization due to the relative proximity of the gastrointestinal tract to the major blood vessels of the medistinum and abdomen. In particular, EUS-guided access of the portal vein (PV) may be favorable given the relative difficulty of PV access via standard percutaneous routes. Potential clinical applications of EUS-guided portal venous access include angiography, measurement of the portosystemic pressure gradient, EUS-guided transhepatic intrahepatic portosystemic shunt (TIPSS) creation and PV sampling for the evaluation in gastrointestinal cancer. The following review will outline the different devices and techniques employed in these

Received: May 8, 2018 Revised: May 21, 2018

Accepted: May 21, 2018

Correspondence: Jason B. Samarasena

Division of Gastroenterology and Hepatology, H. H. Chao Comprehensive Digestive Disease Center, University of California, Irvine Medical Center, 101 The City Drive South, Orange, CA 92868, USA

Tel: +1-714-456-6745, Fax: +1-714-456-7520, E-mail: jsamaras@uci.edu

ORCID: https://orcid.org/0000-0002-2981-3078

cc This is an Open Access article distributed under the terms of the Creative Commons Attribution Non-Commercial License (http://creativecommons.org/ licenses/by-nc/3.0) which permits unrestricted non-commercial use, distribution, and reproduction in any medium, provided the original work is properly cited. applications. Ease of access, safety, and important lessons learned from each approach will be highlighted.

\section{EUS-GUIDED PORTAL VENOUS ANGIOGRAPHY}

The PV is well seen from both the stomach and the duodenum during EUS. The vessel itself is usually in very close proximity to the tip of the echoendoscope, making this an ideal target for vascular access. Portal venous angiography is a modality to assess the anatomy of the hepatic vasculature. Initial cases of successful in vivo EUS-guided PV catheterization were performed in porcine models. In 2004, Lai and colleagues reported an EUS-guided transduodenal approach to access the extrahepatic PV in 21 swine with a $22 \mathrm{G}$ fine needle aspiration (FNA) needle. ${ }^{1}$ A small amount of contrast was injected through the needle for fluoroscopic confirmation of proper placement. ${ }^{1}$ This study proved the feasabilty on a technical level of EUS-guided PV access.

The first study solely assessing PV angiography was a porcine study reported in 2007 by Magno and colleagues. ${ }^{2} 19 \mathrm{G}$, $22 \mathrm{G}$, and $25 \mathrm{G}$ needles were inserted under EUS guidance into the celiac, splenic, superior mesenteric artery, the thoracic and 
abdominal aorta, and the splenic, portal, and hepatic veins (HVs). All vessels were successfully identified and punctured in 5 of 5 pigs. No signs of intraprocedural hemodynamic instability were observed. Immediate postprocedure necropsy showed no signs of injury with the $25 \mathrm{G}$ needle. The $22 \mathrm{G}$ needle left puncture marks without bleeding, and the $19 \mathrm{G}$ needle caused a vascular hematoma in large-caliber vessels with intraabdominal bleeding in 1 of the 5 pigs. Injection of contrast provided good opacification of smaller vessels-the celiac trunk, splenic artery, and HVs-with only transient opacification in larger caliber vessels. As would be expected, the amount of resistance associated with instilling the iodinated contrast was inversely correlated with needle caliber.

Giday and colleagues attempted EUS-guided PV access in 2007 using a transgastric, transhepatic approach with a $25 \mathrm{G}$ needle and a modified endoscopic retrograde cholangiopancreatography (ERCP) catheter. ${ }^{3}$ This protocol was again performed in 2008 as part of another PV catheterization study. ${ }^{4}$ Angiography was achieved using both standard iodinated contrast and medical grade carbon dioxide $\left(\mathrm{CO}_{2}\right)$. PV catheterization was achieved in 6 of 6 swine in 2007 and 6 of 6 swine in 2008 , and no complications were noted in either study. Necropsy showed no evidence of bleeding, hematoma formation, or liver injury. The transgastric, transhepatic approach is postulated to be safer than the transduodenal approach by allowing for natural tamponade of the needle track by liver parenchyma during withdrawal. ${ }^{3,5}$ The use of $\mathrm{CO}_{2}$ as a contrast medium allowed for better visualization of the PV as well as easier intravascular administration through the small-caliber FNA needle when compared to the viscous iodine-based contrast. These studies as a whole suggested that needle puncture of these vessels would not necessisarily lead to intraabdominal hemorrhage or vascular injury.

The safety of $\mathrm{CO}_{2}$ use has been evaluated in both animals and humans. It is highly soluble and easily cleared by the lungs ${ }^{6}$ and, unlike iodinated contrast, is not associated with nephrotoxicity or increased risk for hepatorenal syndrome. ${ }^{7}$ The current data suggest that combining $\mathrm{CO}_{2}$ with a $25 \mathrm{G}$ needle may allow for easier injection of contrast, adequate visualization of the portal circulation, and possibly decreased risk of needleand contrast-related complications.

\section{EUS-GUIDED PORTAL PRESSURE GRADIENT MEASUREMENT}

Portal hypertension $(\mathrm{PH})$, resulting from increased resistance of hepatic sinusoids to blood flow, is most commonly a complication of liver cirrhosis. The pathogenesis involves alteration of the liver vasculature due to fibrosis as well as increased production of vasoconstrictive mediators relative to endogenous vasodilators. Complications of $\mathrm{PH}$ include esophageal varices, portal hypertensive gastropathy, ascites, and hepatorenal syndrome. Measurement of PH has been useful in determining the stage, progression, and prognosis of cirrhosis in individual patients. Portal pressure gradient (PPG) measurement of $\geq 10 \mathrm{~mm} \mathrm{Hg}$ is associated with development of esophageal varices $^{8}$ and PPG of $\geq 12 \mathrm{~mm} \mathrm{Hg}$ with variceal hemorrhage. Reduction of PPG by $20 \%$ or to below $12 \mathrm{~mm} \mathrm{Hg}$ with pharmacotherapy has been found to decrease risk of future bleeding or rebleeding episodes. ${ }^{10,11}$

Previously, PPG values were obtained directly via either a percutaneous approach or using a transjugular intrahepatic portosystemic shunt. The current standard for evaluation of $\mathrm{PH}$ is indirect measurement of the hepatic venous pressure gradient (HVPG). In this technique, a catheter is inserted into the HV percutaneously via either the jugular or femoral vein. The free hepatic venous pressure is recorded and subtracted from the wedged hepatic venous pressure to determine the HVPG. Both percutaneous PV catheterization and HVPG measurement are invasive procedures and require a high level of technical expertise. Direct PV catheterization has been associated with a high complication rate $\mathrm{e}^{12,13}$ and is not commonly performed. Despite the overall safety profile of HVPG measurement, it is only routinely performed at tertiary medical centers. ${ }^{14,15}$ Furthermore, HVPG has been shown to correlate poorly with directly measured portal pressure in cases of presinusoidal $\mathrm{PH}$, which may be seen in cases of non-cirrhotic portal fibrosis and presinusoidal PH, including PV thrombosis and schistosomiasis. ${ }^{4,16,17}$

\section{Animal studies}

Lai and colleagues were the first to report EUS-guided PV pressure (PVP) measurement in a porcine model. ${ }^{1}$ In a cohort of 21 pigs, a $\mathrm{PH}$ model was generated in 14 animals using polyvinyl alcohol injection and a coagulopathy model generated in 7 animals with heparin administration. A transduodenal EUS approach was used to access the PV in 21 pigs with a 22 G FNA needle and a transabdominal ultrasound-guided transhepatic approach in 14 of 21 pigs via a 22-gauge needle. PVP measurements were obtained in 18 of 21 swine. Minor complications found at necropsy included small subserosal hematomas at the EUS puncture site in all 21 pigs and a $25 \mathrm{~mL}$ blood collection between the liver and duodenum in 1 of 7 anticoagulated pigs. Failure to measure pressures in 3 subjects may have occurred due to thrombosis within the FNA needle. There was a strong correlation between EUS- and transhepatic-measured PVP $(r=0.91)$. The development of hematomas in this study suggests that a transduodenal approach that does not traverse the liver may increase risk of bleeding and there- 
fore an approach traversing through liver parenchyma may be favorable.

In 2007, Giday and colleagues used the transgastric approach with a $19 \mathrm{G}$ needle and modified ERCP catheter to obtain continuous PVP measurement without an echoendoscope in place. ${ }^{3}$ Five of 5 pigs were successfully catheterized, and no hemorrhage or liver injury was noted on necropsy in all subjects despite use of a significantly larger caliber needle. Two of 5 pigs were survived for two weeks and exhibited no signs of adverse events prior to and after necropsy. In a later study, the same group used the same methods to measure fluctuations in PVP and inferior vena cava (IVC) pressures in pigs that underwent common endoscopic procedures: esophagogastroduodenoscopy (EGD), colonoscopy, and ERCP. ${ }^{18} \mathrm{PV}$ and IVC were accessed using a $19 \mathrm{G}$ needle and modified ERCP catheter. Access and pressure measurements of both vessels were achieved in 5 of 5 pigs. Necropsy showed no evidence of injury in all subjects. A threefold increase in PVP was noted between baseline and during ERCP. Values of IVC pressure, as well as of PVP for EGD and colonoscopy, were similar between baseline and procedure time.

Schulman and colleagues demonstrated a novel method of measuring PVP in 2016 using an EUS-guided 22 G needle through which a wire with a digital pressure sensor was passed. ${ }^{19}$ Conventional transjugular catheterization was performed as a control. Successful device placement and PVP measurement were achieved in 5 of 5 pigs with no hemorrhage or thrombosis noted on both EUS and post-procedural necropsy. Comparison of EUS-measured PVP with transjugular HVPG measurements showed a difference of within $1 \mathrm{~mm} \mathrm{Hg}$ for all pigs. The study endoscopists rated the procedure as having overall low subjective workload. The authors used the same device to perform PVP measurement in 5 other pigs that were then survived for 14 days before necropsy. ${ }^{20}$ PVP was again measured on day 14. No signs of complications were observed during the 2-week survival period, and necropsy again showed no abnormalities. PVP values on day 0 and day 14 were similar for all 5 pigs.

Our group developed a method of EUS-guided portal pressure measurement using a $25 \mathrm{G}$ needle and simple transducer setup. The apparatus for PPG measurement included a linear echoendoscope, a 25 G FNA-needle and a compact manometer (Fig. 1) with non-compressible tubing. ${ }^{21}$ Prior to echoendoscope insertion, the manometer was zeroed at the mid axillary line. Measurements were conducted in the PV and HV and the IVC. When the PV was targeted, manometry was performed via a transgastric, and less often a transduodenal, transhepatic approach and only the intrahepatic portion near the PV bifurcation was accessed. When evaluating the HV, the needle tip was placed $2 \mathrm{~cm}$ distal to the ostia where possible. Needle placement was meticulous to ensure consistency. $1 \mathrm{~mL}$ of heparinized sa-

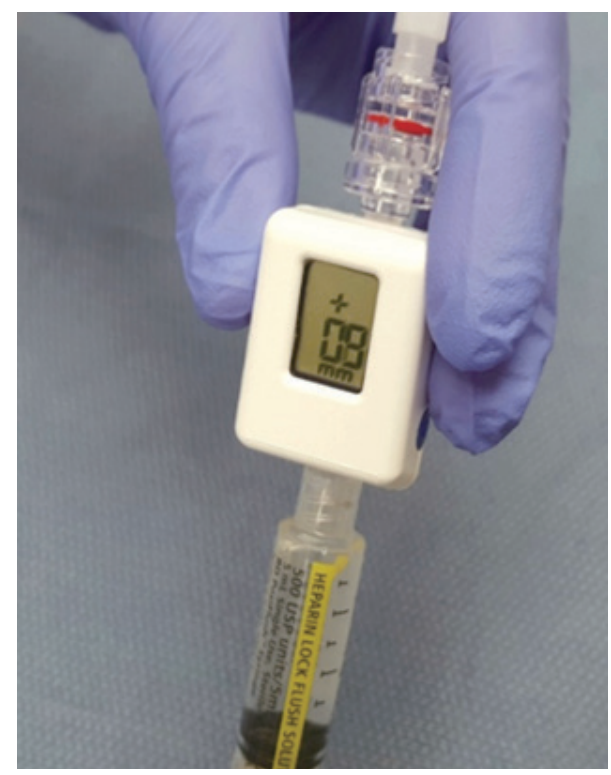

Fig. 1. Compact manometer used for endoscopic ultrasound-guided portal pressure measurement (Cook Medical, Bloomington, IN, USA).

line was flushed through the needle before pressure measurement to clear the needle lumen and confirm intravascular placement. We also measured pressures in a swine model of $\mathrm{PH}$ induced by Dextran-40 administration. Percutaneous measurements in the same vessels were obtained for comparison. All vessels were successfully accessed and pressures measured via EUS in all 3 pigs. Necropsy was not performed, but intraprocedural monitoring showed no signs of cardiorespiratory instability. Correlations between EUS-guided and percutaneous pressure measurements were very strong, with $\mathrm{R}$ values in all vessels greater than or equal to 0.985 .

\section{Human studies}

The first human single case of EUS-guided PVP measurement was reported by Fujii-Lau and colleagues in 2014, in which a 22 G FNA needle connected to an arterial pressure catheter was used to rule out PH in a 27-year-old man with arteriovenous malformations secondary to Noonan syndrome. ${ }^{22}$ The measured PPG was $1 \mathrm{~mm} \mathrm{Hg}$ and correlated with the gradient obtained by interventional radiology at a prior procedure. There was no evidence of bleeding or hemodynamic instability after this procedure. ${ }^{22}$

Our group performed the first prospective pilot study of PPG measurement in human patients with suspected or confirmed cirrhosis. ${ }^{23}$ The set up employed the simple transducer set up discussed above with our animal study. The compact manometer was zeroed at the midaxillary line of each patient, and care was taken to consistently place the needle $2 \mathrm{~cm}$ distal to the HV ostia. Pressure readings were taken of the PV and either the HV or the IVC if anatomy was unfavorable for HV 
access. Needle placement was achieved and PPG measurement obtained in 28 of 28 patients, and no adverse events including bleeding, perforation, or infection were noted. The time required to obtain pressure measurements was short, under 30 minutes per patient. PPG measurements correlated well with clinical and endoscopic parameters with significant differences in PPG noted in patients that were high-risk versus low-risk for cirrhosis and in patients with esophageal varices, portal hypertensive gastropathy, and thrombocytopenia relative to patients without these conditions. There were no complications in any of the 28 patients. In addition, the majority of the patients in this study had EUS-guided liver biopsies performed at the same procedure suggesting that combining a PPG measurement and liver biopsy in the same session should be safe.

\section{EUS-GUIDED PPG MEASUREMENT TECHNIQUE}

The EUS manometry apparatus used in our human study is a simple set up that includes a 25 G FNA needle, non-compressible tubing, a compact digital manometer, and heparinized saline. The tubing is connected by a luer lock to the distal port of

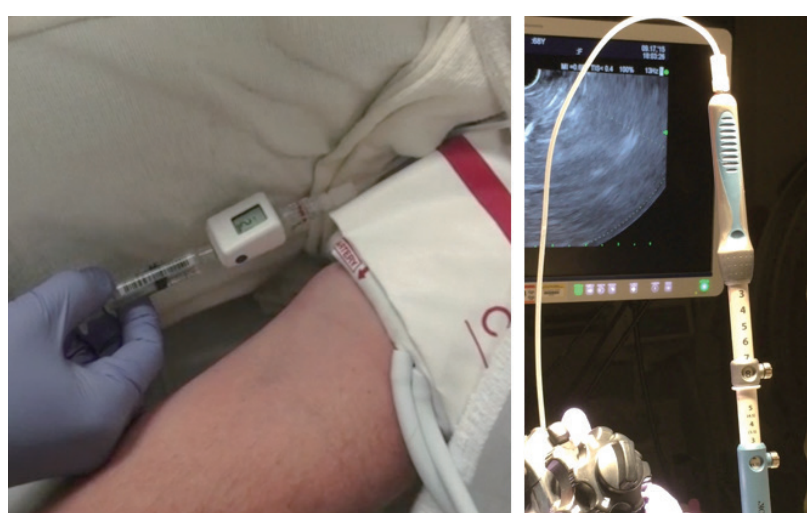

Fig. 2. Endoscopic ultrasound-guided portal pressure measurement apparatus showing non-compressible tubing attached to the fine needle aspiration needle inlet (right pane) and compact manometer being placed at the mid-axillary line of the patient (left pane). the manometer, while the heparinized saline is connected the proximal port. The end of the tubing is connected via a luer lock to the inlet of the $25 \mathrm{G}$ needle. The patient is positioned supine and during EUS-guided pressure measurement reading the manometer is placed at the patient's mid axillary line (Fig. 2). We prefer monitored anesthesia care or general anesthesia for this procedure.

The HV measurement is conducted first. Of the HVs, the middle $\mathrm{HV}$ is targeted most commonly due to its larger caliber and better alignment with the needle trajectory on linear EUS (Fig. 3). Doppler flow is used to confirm the typical multiphasic waveform of hepatic venous flow (Fig. 4). Using the $25 \mathrm{G}$ FNA needle, a transgastric transhepatic approach is used to puncture the HV. Approximately $1 \mathrm{cc}$ of heparinized saline is used to flush the needle which is visible on EUS confirming good position within the vessel. Following the flush, the pressure reading on the manometer will immediately rise and then fall and equilibrate at a steady pressure which is recorded. This measurement should be repeated and second and third time to minimize any error or fluctuation and to give a range of pressures from which to derive a mean pressure. The mean of the three pressures is then considered the HV pressure. The FNA needle is slowly withdrawn from the vein into the liver parenchyma and then back into the needle sheath with Doppler flow on to ensure there is no flow within the needle tract.

The PV measurement is conducted next and the umbilical portion of the left PV is targeted (Fig. 5). Doppler flow is used to confirm the typical venous hum of portal venous flow (Fig. 6). Using the 25 G FNA needle, a transgastric transhepatic approach is used to puncture the PV. The procedure that follows is the same as what was performed for the HV. Approximately $1 \mathrm{cc}$ of heparinized saline is used to flush the needle which is visible on EUS confirming good position within the vessel. Following the flush, the pressure reading on the manometer will immediately rise and then fall and equilibrate at a steady pressure which is recorded. This measurement should be repeated and second and third time. The mean of the three pressures is then considered the PVP. The FNA needle is slowly withdrawn from the vein into the liver parenchyma and then back into

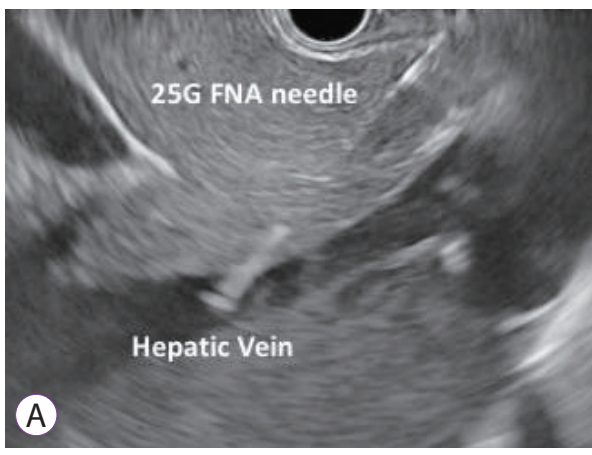

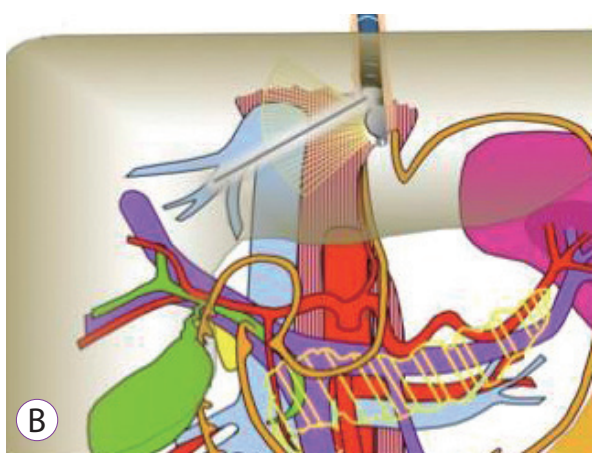

Fig. 3. Endoscopic ultrasound image of needle puncture of middle hepatic vein with $25 \mathrm{G}$ fine needle aspiration needle. 
the needle sheath with Doppler flow on to ensure there is no flow within the needle tract.

The PPG is calculated by subtracting the mean PVP from the mean HV pressure. The patient is recovered in a similar manner to a routine diagnostic EUS with FNA. Post procedural antibiotics are usually given for 5 days post- procedure.

\section{EUS-GUIDED TRANSHEPATIC INTRAHEPATIC PORTOSYSTEMIC SHUNT}

TIPSS is an established treatment of $\mathrm{PH}$ and its complications, mainly for prevention of acute or recurrent esophageal and gastric variceal bleeding and refractory ascites. Buscaglia et al. described the first EUS-guided creation of an intrahepatic portosystemic shunt in a live porcine model. ${ }^{24}$ Under EUS guidance, the $\mathrm{HV}$ and the $\mathrm{PV}$ were sequentially punctured, contrast was injected to confirm needle location within the PV, after which a guidewire was advanced through the needle to the PV, the needle was then removed and a metal stent was inserted over the wire with its distal end in the PV and its proximal end in the HV. There were no complications in all 8 pigs, including

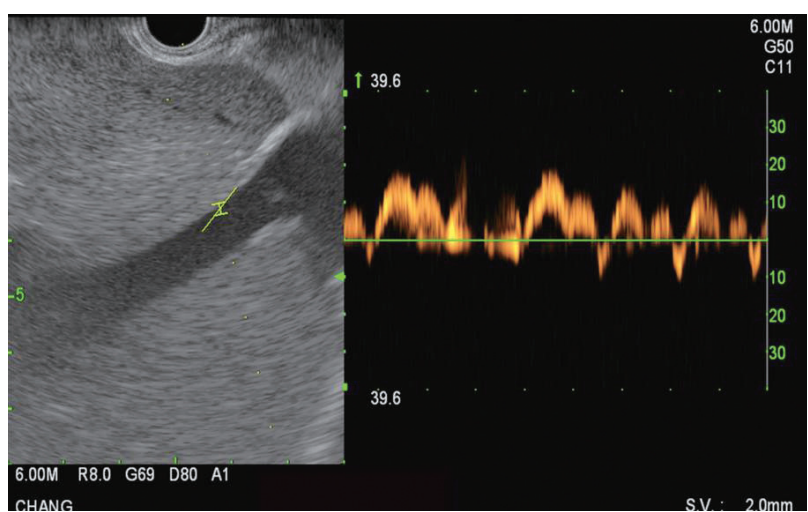

Fig. 4. Endoscopic ultrasound Doppler flow image of middle hepatic vein demonstrating multiphasic waveform. a two-week survival period in two pigs.

Binmoeller et al. used a similar technique to deploy a fully covered lumen-apposing metal stent (LAMS) in a porcine model. ${ }^{25}$ Necropsy confirmed successful stent placement between the PV and the HV with no tissue injury or hematomas. Schulman et al. successfully deployed a LAMS for creation of TIPSS in five pigs. ${ }^{26}$ The HV or IVC was identified using a linear-array echoendoscope and accessed with a 19-G FNA needle preloaded with a digital pressure wire. Mean pressure was recorded. The needle was advanced into the PV, where pressure measurements were again taken, and ultimately exchanged over a guidewire. A LAMS was deployed under EUS and fluoroscopic guidance, with distal and proximal ends positioned inside the PV and HV (IVC), respectively. Stent dilation was performed, and pressure measurements repeated. Animals survived 2 weeks before necropsy Placement of LAMS addressed the concern of stent migration. Technical success was $100 \%$, with no bleeding on necropsy, but two pigs developing partial in-stent thrombosis. Further long-term studies, with refinements of devices and stents, are required before these procedures can be implemented in humans.

\section{EUS-GUIDED PORTAL VEIN SAMPLING FOR GASTROINTESTINAL CANCER}

Distant metastases are responsible for approximately $90 \%$ of cancer-related mortality. ${ }^{27}$ The current understanding of the pathophysiology for risk of developing metastatic disease is linked to the proportion to the amount of shed intravascular tumor cell material. Even small tumors can shed millions of cancer cells by the time a tumor is diagnosed. ${ }^{28,29}$ Current radiologic imaging is limited in its ability to identify micrometastatic $(<2 \mathrm{~mm})$ disease. ${ }^{30}$ Thus, there remains a clinical need not only for the early diagnosis, but for appropriate cancer staging to determine risk of recurrence and to determine who would benefit from neo-adjuvant chemotherapy versus upfront surgery. In line with the concept and safety profile as islet cell

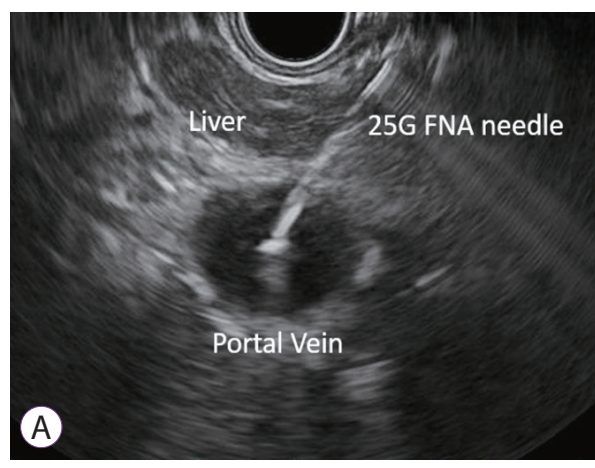

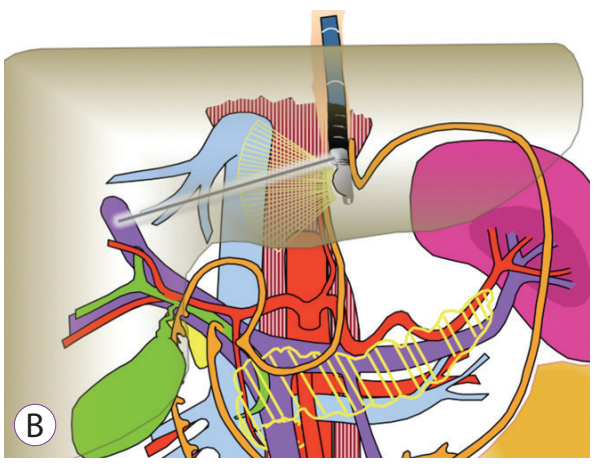

Fig. 5. Endoscopic ultrasound image of needle puncture of left portal vein with $25 \mathrm{G}$ fine needle aspiration needle. 


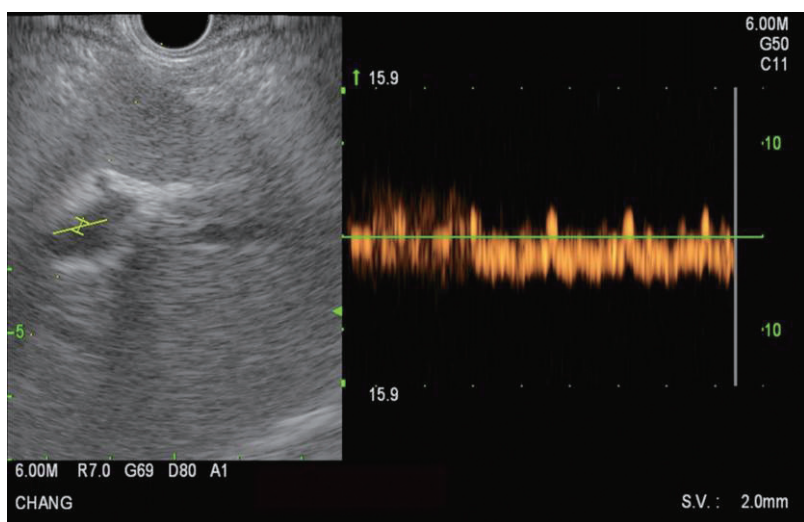

Fig. 6. Endoscopic ultrasound Doppler flow image of left portal vein demonstrating typical waveform.

transplantation by interventional radiology in which the PV is accessed percutaneously, ${ }^{31}$ EUS can provide minimally invasive access to the PV for the purposes of blood sampling. This following will discuss the rationale and technical aspects of EUS-guided PV sampling for diagnostic purposes in gastrointestinal cancer.

In 2015, a study by Catenacci et al. demonstrated that in pancreatic cancer patients, blood could safely be obtained by EUS from the PV and that portal venous blood yields a higher number of circulating tumor cells (CTCs) when compared to peripheral blood. ${ }^{32}$ Using an EpCAM based CTC enrichment method (CellSearch System), CTCs were detected in the PV samples from $100 \%$ of the patients with pancreaticobiliary cancer, but less than $25 \%$ were detected in matched peripheral blood samples. Furthermore quantitatively, there were significantly more CTCs in the portal venous blood compared to peripheral blood (118 CTCs per $7.5 \mathrm{~mL}$ vs. less than 1 CTC per $7.5 \mathrm{~mL}$ ). Specifically, in patients with non-metastatic, borderline resectable cancer, there was a high number of PV CTCs (83.2 per $7.5 \mathrm{~mL}$ ) but less CTCs than in patients with unresectable cancer ( 157.9 per $7.5 \mathrm{~mL}, p=0.23$ ). In the 18 patients in this study, there were no episodes of immediate or delayed post-procedural bleeding or perforation.

With regards to technique in the above study, EUS for PV sampling was performed using a $19 \mathrm{G}$ EUS-FNA needle with a transgastric or transduodenal transhepatic approach. Under EUS guidance, the left and right PVs were identified. After verifying flow signal by foppler, the EUS-FNA needle was advanced transhepatically into the PV and 2-4 aliquots of blood were aspirated and placed in cell preparation tubes (catalog number: 02-685-125, Vacutainer Glass Mononuclear Cell Preparation Tubes; BD, Franklin Lakes, NJ, USA) and CellSave Preservative Tubes (cat: 7900005; Janssen Diagnostics, LLC, Raritan, NJ, USA). Specifically, once the EUS-FNA needle had accessed the PV, the stylet was removed and a $10 \mathrm{~mL}$ negative suction syringe was applied to the FNA needle. Blood was aspirated up the shaft of the EUS-FNA needle into the negative suction syringe. Immediately after aspirating 8 to $10 \mathrm{~mL}$ of blood, a second pre-prepared negative suction syringe to aspirate a second 8 to $10 \mathrm{~mL}$ volume was applied. The puncture site was monitored under EUS for complications. Routine peripheral blood samples were obtained before EUS in parallel and processed identically. To help lower the risk of developing blood clots in the aspirated blood sample, the authors recommend immediately transferring from the negative suction syringe into vacutainer tube containing cell preservatives compatible with downstream application.

The technique of EUS-guided PV sampling appears to be safe and feasible. Future prospective studies will define the role of PV CTCs as prognostic and/or predictive biomarkers in the perioperative setting.

\section{CONCLUSIONS}

Given the wide availability of EUS, an EUS-guided approach for the measurement of the PPG would be a great advance in the field of Endo-Hepatology. PV sampling for the diagnostic work up of pancreaticobiliary malignancy will likely have an important future role in prognostication and staging. The current literature suggests EUS-guided measurement of the PPG and EUS-guided sampling of the PV is safe and feasible. With further work in the area of EUS specific vascular access technologies, the diagnostic and therapeutic opportunities with EUS-guided PV access will continue to expand and likely become a standard component to our current diagnostic evaluation.

\section{Conflicts of Interest}

The authors have no financial conflicts of interest.

\section{REFERENCES}

1. Lai L, Poneros J, Santilli J, Brugge W. EUS-guided portal vein catheterization and pressure measurement in an animal model: a pilot study of feasibility. Gastrointest Endosc 2004;59:280-283.

2. Magno P, Ko CW, Buscaglia JM, et al. EUS-guided angiography: a novel approach to diagnostic and therapeutic interventions in the vascular system. Gastrointest Endosc 2007;66:587-591.

3. Giday SA, Ko CW, Clarke JO, et al. EUS-guided portal vein carbon dioxide angiography: a pilot study in a porcine model. Gastrointest Endosc 2007;66:814-819.

4. Giday SA, Clarke JO, Buscaglia JM, et al. EUS-guided portal vein catheterization: a promising novel approach for portal angiography and portal vein pressure measurements. Gastrointest Endosc 2008;67:338-342.

5. Brugge WR. EUS is an important new tool for accessing the portal vein. Gastrointest Endosc 2008;67:343-344.

6. Hawkins IF, Caridi JG. Carbon dioxide $\left(\mathrm{CO}_{2}\right)$ digital subtraction angiography: 26-year experience at the University of Florida. Eur Radiol 1998;8:391-402. 
7. Liss $\mathrm{P}$, Eklöf $\mathrm{H}$, Hellberg $\mathrm{O}$, et al. Renal effects of $\mathrm{CO}_{2}$ and iodinated contrast media in patients undergoing renovascular intervention: a prospective, randomized study. J Vasc Interv Radiol 2005;16:57-65.

8. Bosch J, Garcia-Pagán JC, Berzigotti A, Abraldes JG. Measurement of portal pressure and its role in the management of chronic liver disease. Semin Liver Dis 2006;26:348-362.

9. Groszmann RJ, Bosch J, Grace ND, et al. Hemodynamic events in a prospective randomized trial of propranolol versus placebo in the prevention of a first variceal hemorrhage. Gastroenterology 1990;99:1401-1407.

10. Albillos A, Bañares R, González M, et al. Value of the hepatic venous pressure gradient to monitor drug therapy for portal hypertension: a meta-analysis. Am J Gastroenterol 2007;102:1116-1126.

11. D’Amico G, Garcia-Pagan JC, Luca A, Bosch J. Hepatic vein pressure gradient reduction and prevention of variceal bleeding in cirrhosis: a systematic review. Gastroenterology 2006;131:1611-1624.

12. Perelló A, Escorsell A, Bru C, et al. Wedged hepatic venous pressure adequately reflects portal pressure in hepatitis $\mathrm{C}$ virus-related cirrhosis. Hepatology 1999;30:1393-1397.

13. Tsushima Y, Koizumi J, Yokoyama H, Takeda A, Kusano S. Evaluation of portal pressure by splenic perfusion measurement using dynamic CT. AJR Am J Roentgenol 1998;170:153-155.

14. Suk KT. Hepatic venous pressure gradient: clinical use in chronic liver disease. Clin Mol Hepatol 2014;20:6-14.

15. Thalheimer U, Bellis L, Puoti C, Burroughs AK. Should we routinely measure portal pressure in patients with cirrhosis, using hepatic venous pressure gradient (HVPG) as a guide for prophylaxis and therapy of bleeding and rebleeding? No. Eur J Intern Med 2011;22:5-7.

16. Sarin SK, Khanna R. Non-cirrhotic portal hypertension. Clin Liver Dis 2014;18:451-476.

17. Pomier-Layrargues G, Kusielewicz D, Willems B, et al. Presinusoidal portal hypertension in non-alcoholic cirrhosis. Hepatology 1985;5:415-418.

18. Buscaglia JM, Shin EJ, Clarke JO, et al. Endoscopic retrograde cholangiopancreatography, but not esophagogastroduodenoscopy or colonoscopy, significantly increases portal venous pressure: direct portal pressure measurements through endoscopic ultrasound-guided cannulation. Endoscopy 2008;40:670-674.

19. Schulman AR, Thompson CC, Ryou M. EUS-guided portal pressure measurement using a digital pressure wire with real-time remote display: a novel, minimally invasive technique for direct measurement in an animal model. Gastrointest Endosc 2016;83:817-820.

20. Schulman AR, Thompson CC, Ryou M. Endoscopic ultrasound-guided direct portal pressure measurement using a digital pressure wire with real-time remote display: a survival study. J Laparoendosc Adv Surg Tech A 2017;27:1051-1054

21. Huang JY, Samarasena JB, Tsujino T, Chang KJ. EUS-guided portal pressure gradient measurement with a novel 25-gauge needle device versus standard transjugular approach: a comparison animal study. Gastrointest Endosc 2016;84:358-362.

22. Fujii-Lau LL, Leise MD, Kamath PS, Gleeson FC, Levy MJ. Endoscopic ultrasound-guided portal-systemic pressure gradient measurement. Endoscopy 2014;46 Suppl 1 UCTN:E654-E656.

23. Huang JY, Samarasena JB, Tsujino T, et al. EUS-guided portal pressure gradient measurement with a simple novel device: a human pilot study. Gastrointest Endosc 2017;85:996-1001.

24. Buscaglia JM, Dray X, Shin EJ, et al. A new alternative for a transjugular intrahepatic portosystemic shunt: EUS-guided creation of an intrahepatic portosystemic shunt (with video). Gastrointest Endosc 2009;69:941947.

25. Binmoeller KF, Shah JN. EUS-guided transgastric intrahepatic portosystemic shunt using the Axios stent. Gastrointest Endosc 2011;73(Suppl 4):AB167.

26. Schulman AR, Ryou M, Aihara $\mathrm{H}$, et al. EUS-guided intrahepatic portosystemic shunt with direct portal pressure measurements: a novel alternative to transjugular intrahepatic portosystemic shunting. Gastrointest Endosc 2017;85:243-247.

27. Esmaeilsabzali H, Beischlag TV, Cox ME, Parameswaran AM, Park EJ. Detection and isolation of circulating tumor cells: principles and methods. Biotechnol Adv 2013;31:1063-1084

28. Mizuno N, Kato Y, Izumi Y, Irimura T, Sugiyama Y. Importance of hepatic first-pass removal in metastasis of colon carcinoma cells. J Hepatol 1998;28:865-877.

29. Massague J, Obenauf AC. Metastatic colonization by circulating tumour cells. Nature 2016;529:298-306.

30. Zhou Z, Qutaish M, Han Z, et al. MRI detection of breast cancer micrometastases with a fibronectin-targeting contrast agent. Nat Commun 2015;6:7984.

31. Funaki B. Islet cell transplantation. Semin Intervent Radiol 2006;23:295297

32. Catenacci DV, Chapman CG, Xu P, et al. Acquisition of portal venous circulating tumor cells from patients with pancreaticobiliary cancers by endoscopic ultrasound. Gastroenterology 2015;149:1794-1803.e4. 